\title{
AGE AND ATTENTIONAL CAPACITY
}

\author{
Dong-Yuan Debbie Wang and Scott Entsminger \\ University of North Florida \\ Jacksonville, Florida USA \\ Email: dwang@unf.edu
}

\begin{abstract}
Summary: Accident and fatality rates begin to increase after age 55. Previous research indicated only weak relationships between crash involvement and poor acuity. The other factor that may influence driving performance is impaired attentional function. Green and Bavelier (2003) showed that action-video-game players have greater attention capacity than non-video-game players. More important, non-video-game players can be trained to enhance the capacity of visual attention and its spatial distribution. In that study, all participants were young adults (aged from 18 to 23). It is not clear whether the reduced driving capability of older adults is due to a decreased attention capacity. In this paper, attention capacity of young and older drivers was examined using a flanker task paradigm. Participants were asked to respond to two shapes (diamond/square) in one of six circles arranged in a ring. At the same time, a distractor (a square/a diamond) was displayed on the left or the right of the ring. The workload of the task was manipulated by presenting different shapes in all the other circles or only one shape was present in the ring. The influence of the irrelevant shape on the performance (flanker effect) under different load conditions was compared between older and younger drivers. Consistent with previous research, the reaction time under the high-load condition was longer than in the low-load condition and older drivers' reaction time was slower than younger drivers'. However, for both age groups, flanker effect only existed for the low-load condition and tends to disappear in the high-load condition.
\end{abstract}

\section{INTRODUCTION}

Investigations of the driving abilities of elderly drivers have focused on the visual impairments as age and/or cognitive problems (Underwood, Phelps, Wright, Loon, \& Galpin, 2005). Older adults tend to suffer more visual impairment as they age. Visual impairment has a direct consequence on driving ability; Owsly et al. (1991) showed that accident risk increased by the prevalence of cataracts and visual attention disorders. However, only weak relationships have sometimes been reported between crash involvement and poor visual acuity. The other factor that may influence driving performance is impaired attentional function (Anstey, Wood, Lord, \& Walker, 2004; Parasuraman \& Nestor, 1991). More importantly, even when there are no signs of visual deficits, attentional deficits can jeopardize driving performance and result in accidents.

Green and Bavelier (2003), in an article published in Nature, showed that action-video-game playing is capable of altering a range of visual skills. They first tested attentional capacity between action-video-game players versus non-video-game players with a flanker compatibility effect (a task that is used to measure the effect of a to-be-ignored distractor stimulus on a target task). In that study, participants were asked to respond to a shape in one of the six circles arranged in a ring. The distractor was presented on either the left or right of the ring. The 
distractor shape could be either compatible with the target (for example, the target was a diamond and the distractor was a diamond) or incompatible with the target (for example, the target was a diamond and the distractor was a square). The results indicated that for non-videogame players, the flanker compatibility effect disappeared when the workload was increased, but not for the video-game players. These results have been interpreted as evidence in support of the hypothesis that a greater capacity exists in the video-game players relative to the non-videogame players. Rosser Jr. et al. (2007) demonstrated that video game play does indeed correlate with laparoscopic surgery skills, and training with video game can enhance performance of surgeons that incorporate laparoscopic suturing skills.

Green and Bavelier trained non-video-game players to play video games for 10 days, and tested participant's visual attention after the training. The results from several visual attention tasks indicated that performance has been enhanced after training. One such test is the useful field of view (UFOV) test. The UFOV is a measure of visual attention, refers to a "functional" spatial area and information in the area can be meaningfully processed at a given time. For example, spotting pedestrians, other motorists on driver's peripheral area. Ball and her colleagues examined the relationship between the UFOV reductions and accidents rate (Ball, Clay, Wadley, \& Roth, 2005; Ball \& Rebok, 1994; Ball, Owsley, Sloane, Roenker, \& Bruni, 1993; Ball \& Owsly, 1991; Ball, Beard, Roenker, Miller, \& Griggs, 1988). Ball et al. (1993) indicated that the UFOV is the most sensitive predictor for crash problems compared to eye health status, visual sensory function, and cognitive status. More important, in that study, Ball et al. showed that UFOV is the strongest index to discriminate between crash-involved versus crash-free drivers: Older drivers (aged 55-90) with a significant reduction in the UFOV were six times more likely to be involved in one or more accident. It has been suggested to use UFOV as a predictor of driving performance (Vance, et al., 2006; Roenker et al. 2003). These results provide a new approach for treating the reduced visual-attentional capability of certain young adults as well as older adults by means of training.

However, all participants in Green and Bavelier's study were young adults (aged between 1823). It is not clear whether the reduced driving capability of older adults was due to a decreased attentional capacity. In this paper, we adapted a paradigm developed by Green \& Bavelier (2003) and Proksch \& Bavelier (2002) to assess the extent of attention capacity of young and older drivers. We compared performance between younger and older non-video-game players in the task. If there was a similar flanker effect as young non-video-game players demonstrated in previous study, it implies that older adults' decreased driving capability may also be due to the reduced attentional capacity. And more importantly, older adults may benefit from training by playing video games.

\section{METHOD}

\section{Subjects}

Eleven undergraduate students, aged from18-23, from the University of North Florida (UNF), participated to earn research credit. Eight older adults ( $>=50$ years old, range from $50-62)$ from UNF community were recruited to participate in this study. 


\section{Apparatus and Stimuli}

Stimuli were presented on a 17" Dell monitor from a Dell computer with E-Prime 1.2. (Psychology Software Tools, Inc.) The visual display contains six circles arranged in a ring in the center of the view and peripheral shape (distractor: a diamond or a square) displayed on the left or the right of the ring. Participants were asked to respond as quickly and as accurately as possible to two shapes (diamond or square) in one of six circles arranged in a ring. The workload of the task is manipulated by presenting different shapes in all the other circles (high-load) or only one shape was present in the ring (low-load). The target shape in the circle subtended an average of $0.6^{\circ}$ vertically and $0.4^{\circ}$ horizontally. The six circles arranged around a center fixation point at a distance of $2.1^{\circ}$. The peripheral shape was placed about $4.2^{\circ}$ from the fixation on the left or the right. The distractor shape could be either compatible with the target (for example, the target was a diamond and the distractor was a diamond) or incompatible with the target (for example, the target was a diamond and the distractor was a square).

\section{Procedure}

Participants were prescreened for this study for safety. If they had a history of epilepsy, motion sickness, attention-related disorder, or migraines, they were excluded from this study. Then they filled in the research questionnaire which includes questions about age, gender and whether they play video games often (if they play video game $>=5$ hours/week, they were classified as videogame players; otherwise, they were classified as non-video game players). Participants were screened based on visual acuity, i.e., their relative eyesight (e.g. 20/20, 20/40). If vision was not $20 / 20$, the participants were asked if they wore corrective lenses so that corrected the vision was $20 / 20$ or better. If not, the participants were excluded from further testing. Then they were asked to sit in front of the computer about $60 \mathrm{~cm}$ away from the monitor. There were two sessions: training and a test session. Each trial began with a cross-fixation display for 1-sec, and then the stimuli (ring and peripheral shape) were displayed for 100-msec. Participants were asked to respond to two shapes, a diamond or a square in each display by pressing either a " $z$ " key or a "?/" key on the keyboard. After their response, visual feedback was given on the screen for 1.5sec. They were instructed explicitly that the peripheral shape was irrelevant to the task and should be ignored. Participants were allowed to ask any questions during the training session and proceed when they were ready. In the test session, there were 5 blocks, each block comprised of 48 trials. Participants were allowed to have a rest during the blocks and when ready, s/he can resume the study by pressing the space bar on the keyboard.

\section{RESULTS}

Only non-video game players' data were reported in this study. Participant's accuracy lower than $50 \%$ was deleted from the data set (totally, three younger adults and one old adults' data were deleted) and trails with RT longer than $1000 \mathrm{msec}$ was deleted in calculating mean RT for each participant.

We were interested in the age effect on general reaction time (RT) and how the flanker effect changes as you age. The two questions were answered by testing two effects on RT: (a) the age main effect on RT and (b) the difference of flanker compatibility effect between two age groups 
under different workload conditions. A GLM was applied to the RT with workload and mapping between the target and distractor (compatible vs. incompatible) as within-subject variables and age as a between-subject variable.

There was a main effect of age, $F(1,17)=8.58, M S E=27,287, p<.05$, indicating that RT for older group was longer than the younger group $(M s=530 \mathrm{~ms}$ and $643 \mathrm{~ms} ; S D \mathrm{~s}=29 \mathrm{~ms}$ and 24 ms, respectively). There was a main effect of workload, $F(1,17)=173.6, M S E=1005, p<.05$, indicating that RT was faster for low-load condition than in the high-load condition $(\mathrm{Ms}=538$ $\mathrm{ms}$ and $635 \mathrm{~ms} ; S D \mathrm{~s}=21 \mathrm{~ms}$ and $18 \mathrm{~ms}$ ). The flanker compatibility effect was not significant but an interaction between the workload and mapping was, $F(1,17)=4.518, M S E=462, p<.05$, which indicated that there was a mapping effect which was different under different workload conditions - further analysis indicated that there was a mapping effect under the low-load condition but this effect tended to disappear under the high-load condition. The effect under low -load condition was $25 \mathrm{~ms}$ but reduced to $10 \mathrm{~ms}$ under high-load condition, but the three-way interaction with age was not significant.

\section{DISCUSSION}

Consistent with previous research, that RT was longer under high-load condition than low-load condition for both groups. And older driver's RT was longer than young driver's RT. However, for both age groups, flanker effect only existed for low-load condition and tended to disappear in the high-load condition. This implies that older drivers demonstrated a similar selective attentional capability with non-video-game players.

Green and Bavelier (2003) indicated that for young adults, selective attentional skill can be trained by playing action-video-games, and more interesting, after training, the UFOV was enhanced. Their results combined with the result of the current experiment provide a new approach for treating the reduced visual-attentional capability of older adults by means of training. Given the convenience of getting a video game and the enjoyment of playing a game, the video game provides a better solution for age-related reduction in attentional capability. Given the evidence that UFOV can be changed with training, from a cognitive perspective, all the attentional factors resulting in the shrinkage of UFOV can be partially attributed to the reduced attentional resource that are available to process the information from a driving scene. This is revealed by the facts that most accidents occurred in the intersections or making a turns in which the attentional demand is high. Also, the reduction of attentional resources is reflected by the divided-attention subtest of UFOV in which showed that as the load of central vision is high, the peripheral vision suffers (Holmes, Cohen, Haith, \& Morrison, 1977; Roge et al., 2002). This is consistent with Lavie's (1995) conclusion that perceptual load is the major determinant of attention selection/allocation.

Video gaming has become a worldwide phenomenon. People of all ages now play video games for enjoyment and to take their minds off the stresses of the current world state. If training does indeed help, it could be a giant step in keeping our roadways safer. The future project will investigate the effect of a training program for older adults with action-video-games on attentional resources and UFOV. 


\section{ACKNOWLEDGMENT}

We would like to thank Dr. Robert Proctor for his comments on the earlier version of this paper. Also, we thank Erica Jarrett, Kelsey Harness and Rachel Wilhelm for their assistance in conducting the experiment.

\section{REFERENCES}

Anstey, K. J., Wood, J., Lord, S., \& Walker, J. G. (2004). Cognitive, sensory and physical factors enabling driving safety in older adults. Clinical Psychology Review, 25, 45-65.

Ball, K. Clay, O., Wadley, V., \& Roth, D. (2005). Predicting driving performance in older adults with the useful field of view test: A Meta-analysis. Proceedings of the 3rd International Driving Symposium on Human factors in Driver assessment, Training and Vehicle Design.

Ball, K., Owsley, C., (2003). Driving Competence: It's Not a Matter of Age. Journal of the American Geriatrics Society, 51, 1499-1501.

Ball, K. \& Rebok, G. (1994). Evaluating the driving ability of older adults. Journal of Applied gerontology, 13, 20-38.

K Ball, C Owsley, ME Sloane, DL Roenker and JR Bruni (1993). Visual attention problems as a predictor of vehicle crashes in older drivers. Investigative Ophthalmology \& Visual Science, 34, 3110-3123.

Ball, K. \& Owsley, C. (1991). Identifying correlates of accident involvement for the older driver. Human Factors, 33, 583-595.

Ball, K, Beard, B., Roenker, D.,Miller, R., \& Griggs, D. (1988). Age and visual search: expanding the useful field of view. Journal of Optical Society of America, 5, 2210-2219.

Green, C. S. \& Bavelier, D. (2003). Action video game modifies visual selective attention. Nature, 423, 534-537.

Holmes, D. L., Cohen, IC. M., Haith, M. M, \& Morrison, F. J. (1977). Peripheral visual processing. Perception A Psychophysics, 22,571-577.

Lavie, N. (1995). Perceptual load as a necessary condition for selective attention. Journal of Experimental Psychology: Human Perception and Performance, 21, 451-468.

Owsley, C., Ball, K., Sloane, M., Roenker, D, Bruni, J. (1991). Visual/Cognitive correlates of vehicle accidents in older drivers. Psychology and Aging, 6, 403-415

Parasuraman, R. \& Nestor, P. (1991). Attention and driving skills in aging and Alzheimer's disease. Human Factors, 33, 539-557.

Proksch, J. \& Bavelier, D. (2002). Changes in the spatial districution of visual attention after early deafness. Journal of Cognitive Neuroscience, 14, 687-701.

Roenker, D., Cissell, G. M., Ball, K. K., Wadley, V. G., \& Edwards, J. (2003). Speed-ofprocessing and driving simulator training result in improved driving performance. Human Factors, 45, 218-233. 
Roge, J., Pebayle, T., Lambilliotte, E., Sptizenstetter, F., Giselbrecht, D., \& Muzet, A. (2003). Influence of age, speed and duration of monotonous driving task in traffic on the driver's useful visual field. Vision Research, 44, 2737-2744.

Rosser, J. C., Lynch, P. J., Cuddihy, L. C., Gentile, D. A., Klonsky, J., \& Merrell, R. (2007). The impact of video games on training surgeons in the $21^{\text {st }}$ Centrury. Arch Surg, 142, 181-186.

Underwood, G., Phelps, N., Wright, C., van Loon, E., \& Galpin, A. (2005). Eye fixation scanpaths of younger and older drivers in a hazard perception task. Ophthal. Physiolo. Opt., 346-356

Vance, D. E., Roenker, D. L., Cissell, G., M., Edwards, J. D., Wadley, V. G., \& Ball, K. K. (2006). Predictors of driving exposure and avoidance in a field study of older drivers from the state of Maryland. Accident Analysis and Prevention, 38, 823-831. 